\title{
Date Time of Most Recent Angina Pectoris
}

National Cancer Institute

\section{Source}

National Cancer Institute. Date Time of Most Recent Angina Pectoris. NCI Thesaurus. Code C91345.

The date and time of the most recent occurrence of ang ina pectoris. 